\title{
An Empirical Study of Role of Linguistic Contexts in Acquisition of English Idioms by Chinese EFL Learners
}

\author{
Xingjia Chong ${ }^{1, a}$ and Yuqing Huang ${ }^{2, b}$ \\ ${ }^{1}$ Gansu Cultural Translation and Interpreting Center, Lanzhou City University, China,730070 \\ ${ }^{2}$ School of Foreign Languages, Lanzhou City University, China, 730070 \\ achongxj@163.com, b120889216@qq.com
}

Keywords: English idioms, linguistic context, context theory

\begin{abstract}
Many EFL learners can understand the meaning of an individual word, but they often failed to comprehend the whole sentence. On the basis of Nation's theory of context, the researcher conducted idiom tests and questionnaire to the subjects from Lanzhou City University, and found that the correct understanding rate of English idioms without context is lower than $50 \%$, while the correct rate promotes dramatically if the English idioms are offered with context. It confirmed that linguistic context plays a positive role in the understanding of English idioms by Chinese EFL learners, and thus teachers should direct students to pay enough attention to the role contexts play in idiom understanding.
\end{abstract}

\section{Introduction}

Idioms, as an essential part of English vocabulary, reflect the environment, life, history, and culture of the native speakers, and are closely associated with their innermost spirit and feeling [1]. An idiom may be understood as a combination of two or more words which are usually structurally fixed and semantically opaque, and function as a single unit of meaning. It is an established form, one that has been accepted by traditional usage. As such, the component words, word order and meaning of each idiom should be learned as a whole.

As a difficult part of language learning, idiom has its own features and characteristics, Chitra Fernando [2] summarized the most frequently mentioned features of idioms as: compositeness, institutionalization, and semantic opacity. Among the theories on vocabulary learning, incidental learning theory proposed by Krashen [3] has received a lot of attention from the researchers. It claims that vocabulary is best acquired incidentally by guessing meanings of unknown words from context through reading. The process of vocabulary learning is also suitable to idioms learning. Nation [4] proposed, "Incidental learning via guessing from context is the most important of all sources of vocabulary 1earning. " Many professionals support the idea of inferring word meanings from contexts. Hunt [5] suggests introducing vocabulary items in such a way as to allow the student to infer or guess the meanings from the context or illustrations. He believes that students should be encouraged to make intelligent guesses about word meanings. It is important to see learning as involving even small increases in knowledge of a word. Among the scholars who try to define idioms, Mcmordie and Seidl [6] are outstanding because some of their opinions on idiom comprehension are very illuminating. Although their study does not break away from the formal perspective, they notice that many idioms are guessable with the help of common knowledge and context.

Contrary to the traditional view that the meaning of an idiom is unpredictable from their constituent parts, Gibbs [7] claims that idioms are partially analyzable and the analyzability of idioms plays an important role in the online interpretation. 
Based on the above theories, this study was designed with the aim of finding out the role context play in the comprehension of English idioms, and empirical study was done among non-English major college students to collect the data.

\section{Research Design}

\subsection{Subjects}

Participants in the present study are divided into two groups, including 30 non-English major freshmen, and 30 non-English major junior students. The 30 freshmen are numbered from 1 to 30 randomly and named group one. The 30 junior students are also numbered from 1 to 30 randomly and named group two. All those students are highly representative, because they come from different grades with different English levels and different proficiencies.

The 30 non-English major freshmen from Bailie College of Engineering, Lanzhou City University as group one. Most of them on average have six years of English learning experience. Usually, their method of learning English are restricted on reciting texts, memorizing grammar rules and do some related exercise. They have no true context, but English is the mandatory subject in the college entrance exam, so their motivation is high.

The other group is from the same college as group two. They choose English as their second majors. These students have nine years of English learning experience, and most of them have past CET4.

\subsection{Instruments}

The instruments for the present study are two tests and a questionnaire. The tests and questionnaire were designed by the researcher. The first part examines whether the students understand the English idioms with or without context. The second part is about the background of the participants and the use of context. In these two tests, students were asked to choose the correct meaning of English idioms. The materials used for the present study are 30 non-literal English idioms.

The non-literal idioms chosen for the study should comply with the following criteria:

1) The idioms chosen have similar structure and lexical items but have different meanings from a Chinese idiom. For example, pull one's leg and Chinese “拖后腿” are often puzzled by many English learners. In fact, the true meaning of English pull one's leg is "to play a joke with someone", while the Chinese idiom means, "not to support one's action”. So, this kind type of idiom may easily confuse the students.

2) It should be made as sure as possible that the idioms chosen for the research are unknown for the subjects. The unfamiliar English idioms to English learners can avoid the previous impression upon them, as to guarantee the correctness of the design.

Based on these criteria, 38 non-literal idioms are selected from A dictionary of American idioms, 5nd Edition compiled by Makkai et al in 2013[8].

The questionnaire was given to all the students in order to get a general picture of their understanding of the context. The questionnaire includes some basic information of participants, such as: name, sex, grade, and score of CET-4 or CET-6.

\subsection{Procedure of the Study}

\subsubsection{Pilot Study}

Five freshman and five junior students are selected as the pilot subjects. They are of very similar English learning background with the subjects chosen for the ultimate experiment. Because of the fact that context in this research serves as a strategic factor in the process of guessing out the meanings of unknown idioms, the focus of the pilot study is to make sure that context can provide a positive effect on understanding the meaning. 
The pilot study is conducted with one pilot subject each time. First, let the ten subjects do the idiom test without any context, then count the number of correct and wrong answers. Second, let the same subject do the other idioms test with context, and then account the number of the correct and wrong answers. Lastly, the two results are compared and analyzed to check the validity and reliability.

According to the pilot survey, it was found that some idioms are too familiar to them and some of them are old-fashioned or impracticable. Meanwhile, there still some idioms that are too hard for the subjects to work out the meaning even with the contexts were provided. To make the result reliable and repeatable, the researcher adjusted the design of the tests and crossed out these questions and thus there are only 30 idioms used in the tests.

\subsubsection{Procedure of the Study}

The research process contains three stages.

Stage one: Let the subjects choose the meaning of the idioms without any contexts. In the limited time, two groups of subjects (freshmen and junior) are asked to complete the test. However, the idioms are offered without any contextual clues. The comprehension of these idioms' meaning all depends on the subjects' first impression and previous knowledge.

Stage two: Let subjects work out the meaning of the idioms with linguistic context. In the limited time, two groups of subjects are asked to complete the other test, which have the context clues. A detailed instruction was given to the subject to direct them paying good attention to the linguistic context of the idioms, enlightening them on techniques and skills for guessing the meanings of idioms.

Stage three: The evaluation stage. A questionnaire, which inquires about the subjects' views and feelings to context, is accomplished by the subjects.

The two test papers and questionnaire were delivered to 60 subjects who were required to do the quiz at same time. The test was conducted in subjects' classroom during self-teaching time. The writer played the role of examiner. The participants were asked to finish the tests and questionnaire without using any dictionaries, reference books, nor would they have any discussions with their classmates. They must keep quiet as if taking an examination. The participants were cooperative and finished the test and questionnaire with patience. It was finished within 30 minutes. The 60 responses were returned and all were selected as valid.

\subsubsection{Data collection}

There are four phases in the process of data collection:

Phrase one: To avoid the uncertain factor that would affect the comprehension of idioms, the author tells the subjects to choose the option by their own comprehension cautiously.

Phrase two: (Idiom Comprehension Test One) 30 idioms without context are presented at one time. If the idiom is known or supposed to the subject, he / she choose A or B option on the blank paper given to him / her. If the idiom is unknown to the subject, he / she choose $\mathrm{C}$ option on the blank paper given to him / her. Then they hand out the paper. If the answer is correct, subject will get one score. If the answer is incorrect or unknown, he /she will get 0 .

Phrase three: (Idiom Comprehension Test Two) The same idioms with linguistic contextual clues are presented to them at one time with the idiom in bold and the other words normal. If the subjects understand the idioms meaning, he / she choose A or B on the blank paper given to him/ her. If the idiom is still unknown to the subject, he/ she choose $\mathrm{C}$ option on the blank paper given to him/ her. Then they again hand out the paper. If the answer is correct, subject will get one score. If the answer is incorrect or unknown, he /she will get 0 .

Phrase four: The subjects are told the correct meaning of each idiom. After the process, the questionnaire is done with 20 subjects from group one and from group two who are chosen randomly as the new subjects. The questionnaire is to collect feedbacks from the subjects about the role the contexts play in the compression of the idioms. 


\section{Data Analysis and Discussion}

\subsection{Comparison of the Guessing Accuracy of Freshmen and Juniors}

After the tests were scored, the source data were typed into the computer. All the data collected were analyzed by Excel 2007. In order to get a clear conclusion about the function of context, the author made a descriptive statistics.

\begin{tabular}{|c|c|c|c|c|c|c|}
\hline & \multicolumn{3}{|c|}{ Test One } & \multicolumn{3}{c|}{ Test Two } \\
\cline { 2 - 7 } & Correct & Incorrect & Unknown & Correct & Incorrect & Unknown \\
\hline $\begin{array}{c}\text { Average } \\
\text { score }\end{array}$ & 9.6 & 14.6 & 5.8 & 17.2 & 8.4 & 4.4 \\
\hline $\begin{array}{c}\text { Accuracy } \\
\text { Rate }\end{array}$ & $32 \%$ & \multicolumn{2}{|c|}{$68 \%$} & $57 \%$ & \multicolumn{2}{c|}{$42.6 \%$} \\
\hline
\end{tabular}

Table 1 Understanding of Idioms in pre-test and the post-test by freshmen students

Table 1 gives us a general idea about the average level of the freshmen. It can be seen that the Chinese English learners did not have a good comprehension of the English idioms. In Test One, the total score is 30, but the average score was only 9.6. The average incorrect score is 14.6 and the unknown average score is 5.8 . The accuracy rate is only $32 \%$, while the rate of errors is as high as $68 \%$. In Test Two, however, the accuracy rate is almost as two times as Test One. The table roughly get a conclusion that context was helpful for the freshmen students to comprehend the English idioms with the context.

\begin{tabular}{|l|l|l|l|l|l|l|}
\hline & \multicolumn{3}{|c|}{ Test One } & \multicolumn{3}{c|}{ Test Two } \\
\hline & Correct & Incorrect & Unknown & Correct & Incorrect & Unknown \\
\hline $\begin{array}{l}\text { Average } \\
\text { score }\end{array}$ & 14.6 & 9.6 & 5.8 & 25.4 & 3.2 & 1.4 \\
\hline $\begin{array}{l}\text { Accuracy } \\
\text { Rate }\end{array}$ & $48 \%$ & $52 \%$ & $85 \%$ & $15 \%$ & \\
\hline
\end{tabular}

Table 2 Understanding of Idioms in pre-test and the post-test by junior students

Table 2 also gives us a general idea about the junior students' average level of the comprehension of the idioms. In Test One, the total score is 30, but the average correct score just got 14.6. The average incorrect score is 9.6 and the average unknown score is 5.8. The accuracy rate of junior students in Test One is $48 \%$, and, the incorrect rate is $52 \%$. In contrast, in Test Two, the accuracy rate has a great leap after the contextual clues were provided. The table further revealed that students at relatively high level proved to comprehend the English idioms much better with the context.

The two tables together give us a full view of the function of the context for the participants in Test One and Test Two. Though subjects of the two groups are from consistent groups at different English levels, but with the average score of correctness, the rate of accuracy and errors, the role of context in the comprehension of English idioms have been tested.

\section{2 Data from the Questionnaire}

According to the questionnaires, 70\% subjects have the motivation to learn the English but they lack the interest because the English teaching classroom is boring. At the same time, they think that the process of learning English is a little difficult. The subjects' English level is not same. Among the all subjects, 55\% have past the CET-4 and CET-6, and 45\% have not past the exams. Over 75\% subjects always take the old and invariable method to recite the words. 
About $87 \%$ subjects think the situational approach is an effective method to understand the idioms' meaning. No matter what level are they, $76 \%$ subjects prefer to choose context as a new method to study the new idioms. As to the output of idioms, over $80 \%$ students approve of the situation teaching method in which the context is provided and discussed, that would foster their acquisition of the vocabulary.

\subsection{Discussions}

English and Chinese belong to different language families. Therefore, they differ greatly with each other. However, some of English idioms have the similar meaning with the Chinese idioms. There have a lot of English idioms and Chinese idioms that can be not equal to each other. For example, pull one's leg does not mean to be one's obstacle, but means play a joke with someone.

The results of the pre-tests and the post-tests show a change in the students' tendency and effectiveness of using contextual clues to guess meanings when they encountered unknown words in reading. They showed the effectiveness of the context. In Test One the students were not given any context clue. In Test Two, the subjects were instructed to recognize and use different kinds of contextual clues, and they became more conscious of context and felt it more useful. And the results of Test Two showed that their rate of accuracy in idiom understanding improved greatly. Almost all the students in the group comprehend context more deeply and successfully. Therefore, we can firmly say that using context can be further improved if the students keep practicing it in their reading activities.

\section{Conclusions}

Based on the above analyses, some general conclusions can be reached as follows:

Firstly, by analyzing the data elicited by test, it is found that context is more efficiently and plays a important role in comprehend the idioms than the common method (the post-test's correct rate is $57 \%$ and $85 \%$, but the pre-test correct rate only is $32 \%$ and $48 \%$ ). The data shows the effectiveness of the context. And context plays an important role in the comprehension process of idioms. The striking function of context provides a certain environment and culture background for the language speakers. It is important for EFL students to understand the idioms' meaning. From the test of subjects' result and comparison, we can draw a conclusion that the context plays a positive role in understanding English idioms.

Secondly, concerning how to comprehend an unknown non-literal English idiom correctly, one should first correctly figure out what the literal meaning of the non-literal idiom is and try to understand the context correctly. Then, put the literal meanings into the context. With the help of conventional knowledge, conceptual metaphor search, etc., several figurative meanings may pop out based on the literal one.

Finally, from questionnaire, the teacher should lead the right direction before teaching words, phrases or passages. Teachers should pay attention to the application of context in daily teaching. Then, a1l of them will make a great progress in reading after the period of training. Using context to guess idioms' meaning is a useful approach because it encourages readers to make predictions, which is a useful generalized skill. Training students to guess meaning from context gives them a powerful aid to comprehension. So word guessing in context has a positive effect on students' reading comprehension.

\section{References}

[1] Yunfei, Zhang. An Introduction to Modern English Lexicology [M]. Beijing: Beijing Normal University Press, 2004.190.

[2] Chitra Fernando. Idioms and Idiomaticity [M]. New York: Oxford University Press,1996.3. 
[3] Krashen.S. 1983. Cited in Schmid.R.W. The Role of Consciousness in Second Language Learning[J]. Applied Linguistics, 1990 11(2) 129-158.

[4] Nation, LS. P. Teaching and Learning Vocabulary [M]. New York: Newbury House, 1990.232.

[5] Hunt, Alan. Constraints on Inferring Word Meaning from Context [J]. Kansai Gadai University Journal of Inquiry and Research. 199663 239-249.

[6] McMordie, W. \& Seidl, J. English idioms and how to use them [M].New York: Oxford University Press. 1978.

[7] Gibbs R. W. The Poetics of mind [M]. New York: Cambridge University Press.1994.

[8] Makkai, A. et al. A Dictionary of American Idioms[M] (5 ${ }^{\text {th }}$ Edition). New York: Barron's Educational Series, 2013. 